Zavaleta, M. (2014). Coaliciones independientes. Lima: Instituto de Estudios Peruanos.

\title{
Cinco reflexiones sobre el estudio de los regímenes políticos subnacionales ${ }^{+}$
}

\author{
DANIEL ENCINAS* \\ Pontificia Universidad Católica del Perú \\ danielencinaszevallos@gmail.com \\ https://doi.org/10.18800/rcpg.201602.002
}

\section{Resumen}

En los últimos años han proliferado los estudios que resaltan que muchas democracias son territorialmente heterogéneas, porque los atributos mínimos de este tipo de régimen tienen una presencia muy débil o están ausentes a nivel subnacional. Tomando en consideración tanto las publicaciones precedentes como la agenda de investigación pendiente, el presente trabajo plantea cinco reflexiones sobre este campo de estudio: dos son empíricas, otras dos conceptuales y una es teórica. Específicamente, se resalta que este fenómeno ha ocurrido en distintos momentos históricos y lugares del mundo; también se demuestra que la mayoría de investigaciones se ha centrado en países federales pese a que existen razones de peso para extender los estudios a los países unitarios; a continuación, se realiza un balance de las ventajas y desventajas de los diferentes conceptos propuestos; asimismo, se plantea cuáles son las enseñanzas en las estrategias conceptuales que nos deja la literatura sobre la democracia a nivel nacional; y, finalmente, se presentan los debates teóricos en torno a las explicaciones. Para desarrollar estas reflexiones, el artículo se basa en una extensa revisión de literatura y en evidencia empírica proveniente de un trabajo de campo en dos regiones del Perú.

Palabras clave: política subnacional, regímenes políticos, autoritarismo, democracia, federalismo, unitarismo.

\section{Five reflections on the study of subnational political regimes}

\footnotetext{
Abstract

In recent years, studies have shown that many democracies are territorially heterogeneous, because the minimal attributes of this type of regime have a very weak presence or are absent at the subnational level. Taking into account both the previous publications and the pending research agenda, the present paper raises five remarks on this field of study: two are empirical,

* Politólogo por la Pontificia Universidad Católica del Perú (PUCP) y miembro del Grupo de Investigación de Política Subnacional en esa misma casa de estudios, donde tiene experiencia como docente y predocente. Previamente, ha publicado artículos y reseńas en revistas académicas como Latin American Research Review, Revista de Ciencia Politica (Santiago-Chile) y Politai.

+Recibido el 4 de enero de 2017; aceptado el 1 de marzo de 2017.
} 
two are conceptual and one is theoretical. Specifically, it is emphasized that this phenomenon has occurred in different historical moments and places of the world; It is also shown that the majority of research has focused on federal countries despite the fact that there are compelling reasons to extend the studies to the unit countries; Then a balance is made of the advantages and disadvantages of the different proposed concepts; Likewise, what the lessons are in the conceptual strategies left to us by the literature on democracy at the national level; And, theoretically, the theoretical debates about explanations are presented. To develop these re exiones, the article is based on an extensive literature review and empirical evidence from a field work in two regions of Peru.

Key words: Subnational politics, political regimes, authoritarianism, democracy, federalism, unitarianism. 


\section{INTRODUCCIÓN}

El trabajo pionero de Rosa Arévalo (2015) sobre la región peruana de Áncash ha abierto una importante e interesante agenda de investigación sobre regímenes políticos subnacionales en Perú. Aunque el trabajo tenía como objetivo explicar la estabilidad en el poder del entonces presidente regional César Álvarez, uno de los factores señalados como clave (además de la corrupción y el clientelismo) fue el autoritarismo subnacional. De esta manera, brindó evidencia empírica y contribuciones teóricas relevantes para entender este fenómeno fuera de los países federales donde se suponía que el fenómeno estaba confinado.

Sin embargo, todavía es mucho lo que se puede estudiar sobre regímenes políticos en el Perú y otros países unitarios. Las conclusiones a las que se lleguen dialogarán con un campo de estudio vibrante en el que han proliferado las investigaciones durante los últimos años. Por estos motivos, recientemente Carla Cueva, Noelia Chávez, Mariana Ramírez y yo publicamos un documento de trabajo en el que intentábamos resumir los aportes conceptuales y explicativos de la literatura enfocada en América Latina (Cueva, Chávez, Encinas y Ramírez, 2016). A su vez, venimos trabajando en una investigación empírica para explicar el surgimiento, mantenimiento y caída de lo que denominamos un autoritarismo competitivo de nivel subnacional en Áncash (Encinas, Chávez y Cueva, en elaboración).

Sobre esta base, el presente artículo plantea cinco reflexiones sobre el estudio de los regímenes políticos subnacionales, teniendo en mente la agenda de investigación pendiente ${ }^{1}$. Primero, se presentan reflexiones sobre las contribuciones empíricas, relacionadas con el conocimiento substantivo generado sobre casos alrededor del mundo. A continuación, se destaca el cambio reciente en la tendencia de la literatura a asumir que este fenómeno es exclusivo de los países federales. La tercera y cuarta sección están dedicadas al tratamiento de los conceptos. Concretamente, se presentan las ventajas y desventajas de las diferentes alternativas para nombrar el fenómeno; así como los retos compartidos y las enseńanzas que provee la literatura nacional sobre democracia y democratización. La quinta sección se enfoca en las diferentes explicaciones existentes para entender el fenómeno. Finalmente, se cierra el artículo con algunas conclusiones.

\footnotetext{
Estas cinco reflexiones, por lo tanto, provienen del trabajo conjunto con Carla Cueva, Noelia Chávez y Mariana Ramírez. Agradezco a ellas por el aprendizaje logrado en estos ańos de trabajo en equipo.
} 


\section{A pesar de la tercera ola de democratización, La «YUXTAPOSICión DE REGÍMENES» ES UN FENÓMENO EXTENDIDO EN DIVERSOS PAÍSES}

Tradicionalmente, el estudio de los regímenes políticos ha tenido una orientación nacional. Para responder a las interrogantes sobre los tipos y subtipos de regímenes, sus orígenes, estabilidad, funcionamiento y cambios, la literatura asumía que estos fenómenos tenían como locus el Estado-nación. Salvo excepciones como Key (1949) y Dahl (1971), trabajos clásicos como Lipset (1960), Moore (1966), Almond y Verba (1963) y O’Donnell y Schmitter (1986) tienen en común esta característica. Lo que prevalecía era una perspectiva nacional de las instituciones, los actores y los procesos (Gibson, 2005).

La ola global de caída de regímenes autoritarios a partir de fines de la década de 1970 e inicios de la de 1980 continuó con esta tendencia. Gran parte de las publicaciones académicas estuvieron marcadas por el optimismo que generó la extensión de la democracia de un conjunto relativamente pequeño de países económicamente más desarrollados a países fuera de este cuadrante y caracterizados históricamente por autoritarismos duraderos y/o la inestabilidad de cualquier tipo de régimen (Levitsky y Way, 2015). Pronto, sin embargo, el optimismo inicial tuvo que ser matizado ante la evidencia de que los resultados postautoritarios podían derivar en democracias con amplias deficiencias (O’Donnell, 1994), regímenes dudosamente democráticos (Collier y Levitsky, 1997), periodos de constante inestabilidad, retrocesos autoritarios o surgimiento de nuevos tipos de autoritarismos (Levitsky y Way, 2010).

No obstante, debido a la atención centrada en los países como conjuntos homogéneos y no en aquello que sucede en su interior, otro reto de la democratización pasó relativamente inadvertido. Muchas de las nuevas democracias eran, en cierto sentido, superficiales. Si bien generaron un cambio crucial en la manera en que se accedía al poder y se tomaban decisiones en las esferas más importantes, los atributos que caracterizan a este tipo de régimen no necesariamente incidían en niveles de gobierno más bajos que el nacional: estados, provincias, regiones, departamentos, municipalidades, intendencias, etcétera. No fue hasta la década de 1990 que la literatura empezó a incluir de forma más sistemática una orientación subnacional en los estudios sobre democracia y democratización (por ejemplo, O’Donnell, 1993; Fox, 1994; Hagopian, 1996; Snyder, 1999), reconociendo el desarrollo territorialmente desigual de los regímenes políticos. Esta primera generación de trabajos exploró «las tensiones entre los esfuerzos a nivel nacional por consolidar la democracia y 
la persistencia de prácticas políticas no democráticas a nivel local» (Snyder, Moncada y Giraudy, en prensa)2.

Pero en décadas posteriores se ha generado una proliferación de estudios enfocados en esta temática debido al llamado «giro subnacional» de la política comparada (Snyder, Moncada y Giraudy, en prensa). Entre las características más resaltantes de este "giro subnacional» se encuentra el haber buscado evitar las consecuencias teóricas y metodológicas de hacer investigaciones que mantienen un "sesgo del país en su conjunto» (Lijphart, 1971, citado por Snyder, 2001), porque muchos fenómenos políticos y sociales rara vez se despliegan de modo homogéneo a lo largo del territorio. Ocurren, más bien, a través de patrones geográficamente desiguales que generan variaciones al interior de los países (Giraudy, 2012, p. 1). De esta manera, en lugar de hacer un llamado a abandonar los estudios nacionales, se ha hecho énfasis en la necesidad de complementarlos con investigaciones subnacionales o, en determinadas situaciones, priorizar uno u otro tipo de aproximación.

A partir de entonces, la discusión sobre regímenes políticos ha estado fuertemente atraída por estudiar aquello que Gibson (2005) bautizó como «yuxtaposición de regímenes». Una situación en la que las características de la democracia nacional están parcial o mayoritariamente ausentes en alguna unidad subnacional. Así, por ejemplo, el fenómeno estaría presente en el país A si este fuera nacionalmente democrático $\mathrm{y}$, a su vez, la región $\mathrm{X}$ (perteneciente a A) fuese autoritaria. La novedad del fenómeno y la relevancia de estudiarlo radica en que, a partir de la literatura nacional sobre democracia y democratización, se esperaría que tanto $\mathrm{X}$ como otras regiones de $\mathrm{A}$ (digamos $\mathrm{Y}$ o $\mathrm{Z}$ ) fuesen tan democráticas como el país en su conjunto. Obviamente, la situación contraria (autoritarismo nacional y democracia subnacional) también calificaría como una yuxtaposición de regímenes, pero el énfasis de la literatura ha sido el mencionado.

Este fenómeno, no obstante, dista de ser hipotético. Se ha documentado diferentes casos alrededor del mundo que revelan «una distribución desigual en las prácticas y la institucionalización de la democracia al interior del Estadonación» (Gibson, 2005, p. 104). En América Latina, las jurisdicciones subnacionales estudiadas pertenecen principalmente a México, Argentina y Brasil. Concretamente, se han realizado constantes referencias al carácter autoritario de provincias en Argentina como Formosa, La Rioja, Santiago del Estero, San

\footnotetext{
2 De aquí en adelante, todas las citas textuales de títulos que aparecen en inglés en la bibliografía han sido traducidas libremente por el autor.
} 
Luis y Catamarca (Gibson, 2005; Gervasoni, 2011; Giraudy, 2009; Behrend, 2011); en México, a los estados de Oaxaca, Chiapas, Tabasco, Puebla y Veracruz (Fox, 1994; Snyder, 1999; Durazo Herrmann, 2010; Benton, 2012; Giraudy, 2009) y, finalmente, en Brasil, a los estados de Bahia y Maranhão (Montero, 2010). Por ejemplo, en la provincia de San Luis en Argentina, los hermanos Rodriguez Saá son miembros de una familia que participó en la política argentina desde las guerras de Independencia y a partir de la década de 1980 gobernaron autoritariamente la provincia por más de dos décadas; en México, el Partido Revolucionario Institucional (PRI) dominó el estado de Oaxaca no solo durante las siete décadas de hegemonía nacional del partido, sino incluso luego de la transición nacional a la democracia; y, finalmente, en Brasil, Antonio Carlos Magalhaes dominó la política del estado de Bahia desde la década de 1970 hasta el segundo quinquenio de 2000.

Otros países que han sido analizados son Estados Unidos, Filipinas, India, Kirguistán y Rusia, rescatándose que el fenómeno también ocurrió en olas de democratización previas (Rebolledo, 2011; Gibson, 2013; Giraudy, 2015; Behrend y Whitehead, 2016). En otras palabras, en distintos contextos y momentos históricos se ha encontrado que puede existir una democracia a nivel nacional a la par de una o más jurisdicciones subnacionales dudosamente democráticas o que, cabalmente, no cumplen con los requerimientos mínimos de este tipo de régimen. Estos hallazgos de la literatura no solo responden a una preocupación académica sino también (y sobre todo) a un interés normativo: «es éticamente inaceptable que los ciudadanos de un mismo país gocen en diferente medida de los derechos políticos que les garantiza la constitución dependiendo del lugar donde residen» (Gervasoni, 2011).

\section{LA MAYORÍA DE ESTUdIOS SE HA CONCENTRADO EN PAÍSES FEDERALES, PERO EXISTE EVIDENCIA DE QUE SERÍA NECESARIO EXPANDIR EL ANÁLISIS HACIA PAÍSES UNITARIOS}

De lo mencionado previamente, resalta que la mayoría de investigaciones ha estado concentrada en el conjunto de países federales. No se trata de una coincidencia. La centralidad brindada a estas entidades se justifica porque estos países suelen brindar una autonomía más o menos amplia a sus jurisdicciones subnacionales, existe una vigorosa dinámica política en estas unidades, los gobernantes subnacionales suelen ser actores relevantes en la política nacional y/o el autoritarismo subnacional ha sido un fenómeno que se ha mantenido por largos periodos de tiempo. Giraudy (2015) destaca que las cinco provincias que 
califican como no democráticas en Argentina fueron gobernadas ininterrumpidamente por el peronismo desde 1983; mientras que en México, los regímenes controlados por el PAN duraron hasta dieciséis años y algunos controlados por el PRI más de ochenta. Son décadas de estabilidad autoritaria en el nivel subnacional sobreviviendo los procesos de democratización a nivel nacional.

Como consecuencia, Gibson (2005, p. 11) señala que el autoritarismo subnacional «estará limitado en alcance a las provincias (o "estados") en sistemas federales»" Gervasoni (2011, p. 15) plantea que "la extensión en que la ciudadanía de muchas democracias federales disfruta de los beneficios de la libertad política varía [...] al interior de las fronteras nacionales» ${ }^{4}$; y Behrend y Whitehead (2016, p. 3), por su parte, indican que «la variación democrática a nivel subnacional podría ser tan relevante en países unitarios altamente descentralizados como en países federales». A partir de estas afirmaciones, queda claro que gran parte de la literatura tiene como supuesto que un prerrequisito para que ocurra la yuxtaposición de regímenes es ser un país federal o, al menos, parecerlo en la práctica. En palabras de Giraudy: «los trabajos en esta temática han producido teorías cuyas premisas centrales solo pueden encontrarse en federaciones o países altamente descentralizados» (2015, p. 8).

No obstante, considerar que el fenómeno sucede exclusivamente en estos países es problemático por diversos motivos. En primer lugar, deja de lado que el federalismo y el unitarismo no constituyen una dicotomía rígida. El federalismo se define como «una política nacional con dos (o múltiples) niveles de gobierno, cada uno ejerciendo autoridad exclusiva sobre áreas de políticas públicas [...], pero en el que solo un nivel de gobierno —el gobierno centrales internacionalmente soberano» (Gibson, 2004, p. 5); mientras que en el unitarismo existe «un único centro nacional organizador de la vida política, económica y social» (Montecinos, 2007, p. 12). En este sentido, son arreglos constitucionalmente definidos (Falleti, 2005) cuyo correlato real podría variar. Como ha sido evidenciado, muchas veces las instituciones informales importan tanto o más que las formales (O’Donnell, 1996; Helmke y Levitsky, 2006).

En esta misma línea, se asume implícitamente que todos los federalismos son muy similares, pese a que la evidencia empírica demuestra variación en el impacto de las instituciones federales y la dinámica de sus políticas internas. Un trabajo enfocado en América Latina llegó precisamente a esta conclusión comparando Argentina, México, Brasil y Venezuela (Gibson, 2004).

Cursivas agregadas.

Cursivas agregadas. 
Adicionalmente, resulta cuestionable que se ponga énfasis en que un país unitario altamente descentralizado es semejante a uno federal. Por un lado, no se pone atención a que la descentralización, entendida como «un proceso de reforma del estado compuesto por un conjunto de políticas públicas que transfieren recursos, responsabilidades o autoridades de niveles más altos a niveles más bajos de gobierno» (Falletti, 2005, p. 328), aplica tanto a países unitarios como federales. Los niveles de descentralización solo están determinados inicialmente por la estructura del estado (siendo los federalismos más descentralizados que los unitarismos), pero «explica poco los cambios dentro de un país a lo largo del tiempo» (Escobar-Lemmon, 2001, p. 27). De este modo, algunos procesos de descentralización han impactado en mayor medida en un país unitario como Colombia que en uno federal como Argentina (Falletti, 2005).

En el campo de estudio de los regímenes políticos subnacionales se ha demostrado que las diferencias entre los federalismos generan - a su vezdiferentes escenarios de oportunidades y limitaciones. Dependiendo del nivel de constreñimiento que imponen las reglas formales, el autoritarismo subnacional puede configurarse a través de la eliminación de las instituciones democráticas (estableciendo «reglas institucionales» diferentes) o a través de su manipulación o subversión (Gibson, 2013, p. 13). Por ejemplo, en México se configuraron autoritarismos subnacionales a partir de prácticas ilegales e informales porque «la constitución nacional y las regulaciones electorales nacionales limitan significativamente a los sistemas políticos de nivel estatal» (Gibson 2013, p. 15) 5 . Esto podría ser muy cercano a lo que ocurre en países unitarios donde se restringe la autonomía subnacional para escribir reglas de juego propias. En otras palabras, para algunas características, la distancia entre un país unitario y uno federal podría ser menor a la existente entre dos países federales.

Por último, resulta importante considerar la evidencia disponible hasta el momento. Eaton y Prieto (en prensa) documentan que en Colombia también ocurrió una yuxtaposición de regímenes, pese a no ser un país federal. Los autores plantean una comparación entre las jurisdicciones de Cesar y Magdalena, donde existe una trayectoria divergente en el autoritarismo subnacional, pese a similitudes políticas, económicas, estructurales y en el dominio de la misma facción militar. Por su parte, Sidel (2014) ha contribuido con evidencia sobre la presencia de autoritarismos subnacionales en Filipinas, otro país unitario, a partir del contraste de las provincias de Cebu y Cavite. De acuerdo con Behrend 
y Whitehead (2016a), el autor «muestra cuánta relevancia tiene el control económico local para el desarrollo de estructuras iliberales subnacionales [...] [y] cómo en muchas regiones de América Latina las familias políticas juegan un importante rol».

Otro caso de autoritarismo subnacional en un país unitario es la región peruana de Áncash durante los años en que César Álvarez estuvo en el poder (2007-2014). Los trabajos de Arévalo (2015) y Cueva et al. (2016) demostraron las similitudes del régimen surgido en este caso con otros en países como México y Argentina. Esta autoridad subnacional se las arregló para crear una oficina de inteligencia paralegal, apodada "La Centralita», donde se pactaban millonarios acuerdos de corrupción, se controlaban muchos de los medios de comunicación locales y se tomaban decisiones como encargar la persecución y asesinato de sus detractores a sicarios que trabajaban constantemente a sus órdenes. Como consecuencia, durante estos años los medios independientes fueron marginales, las libertades civiles básicas de la población no estuvieron garantizadas, y muchos opositores políticos y de la sociedad civil vivieron permanentemente amenazados o directamente reprimidos. Pese a que las elecciones continuaron siendo el principal medio de acceso al poder, estas características alejan a Áncash de los estándares que debería cumplir una democracia. Puede calificarse como un autoritarismo competitivo de nivel subnacional (Cueva et al., 2016).

En conclusión, existe un nuevo conjunto de casos que debe ser considerado en el análisis. Esto ocasiona que sea necesario reconsiderar los conceptos que se utilizan para describir el fenómeno, porque podrían no aplicar a países unitarios. También es importante ampliar el abanico de preguntas que se realizan: más allá de la tradicional interrogante sobre la estabilidad de los regímenes políticos subnacionales, importará entender por qué algunos duran más que otros, cuáles son las causas de sus orígenes y eventual caída, por qué hay diferentes niveles de vulneración de los atributos básicos de la democracia, cuáles son los tipos de regímenes políticos subnacionales, entre otras. En este sentido, las conclusiones teóricas podrán ser revisadas a la luz de la nueva evidencia empírica que se genere. 


\section{LA LiTERATURA SIGUE DISCUTIENDO CUÁLES SON LAS VENTAJAS Y DESVENTAJAS DE LOS DISTINTOS CONCEPTOS QUE HAN SIDO PROPUESTOS PARA DESCRIBIR EL FENÓMENO}

Hasta hace algunos años, las discusiones conceptuales estaban prácticamente ausentes de la literatura sobre regímenes políticos subnacionales. Se hacía referencia a «enclaves autoritarios» (Fox, 1994) o "autoritarismos subnacionales» (Gibson, 2005) sin suficiente precisión sobre qué implicaban estas etiquetas. Como indicó Giraudy, muchos trabajos no ofrecían «una conceptualización clara de cómo conciben los regímenes políticos, mucho menos una discusión sobre sus dimensiones, e indicadores» (2012, p. 5). De este modo, se estaba dificultando la prueba de hipótesis y la construcción teórica, porque la función principal de las definiciones es establecer fronteras nítidas entre aquellos casos que abarca un concepto y aquellos que no (Sartori, 1970).

Posteriormente, la calificación de autoritarismo subnacional empezó a ser cuestionada. Según la definición clásica de Linz, los autoritarismos son regímenes políticos:

[...] con un pluralismo político limitado y no responsable, sin una elaborada ideología rectora pero con mentalidades distintivas, sin movilización política intensiva o extensiva excepto en ciertos estadios de su desarrollo, y con un líder u ocasionalmente un pequeño grupo ejerciendo el poder dentro de límites formales pobremente definidos pero ciertamente bastante predecibles $(1964$, p. 255) .

El objetivo explícito del autor fue generar un contraste con la democracia que, a su vez, mantuviera distancia del totalitarismo. No obstante, posteriormente se ha reconocido que los «diferentes tipos de autoritarismos difieren tanto entre sí como de la democracia» (Geddes, 1999, p. 121). Los subtipos identificados son, entre otros, las monarquías, los regímenes militares, los regímenes personalistas y los regímenes de partido único. Difícilmente aplicarían al nivel subnacional.

Diversos autores concuerdan que el concepto es demasiado «intenso» para hacer referencia a una unidad subnacional que pertenece a un país con una democracia a nivel nacional que genera constreñimientos para el abuso de poder (Gervasoni, 2011). También se señaló la imprecisión de considerar que prácticas como el clientelismo, la corrupción, el nepotismo y el patronazgo solo ocurrían bajo autoritarismos, cuando estos fenómenos pueden encontrarse en diversas democracias. Asimismo, se hizo énfasis en que el concepto de

\footnotetext{
Traducción propia.

Traducción propia.
} 
régimen político hacía referencia a reglas sistemáticas, no a hechos aislados que no implican una vulneración a las características mínimas de una democracia (Behrend, 2012).

Los intentos por solucionar estas deficiencias derivaron en la creación de nuevos conceptos, pero muchos de estos no se diferencian claramente entre sí. Se ha señalado la existencia de "niveles de democratización subnacional» (Tuñón, 2007), «regímenes no democráticos» (Giraudy, 2015), «regímenes híbridos»(Gervasoni, 2011), «juegos cerrados» (Behrend, 2011), «prácticas iliberales» (Behrend y Whitehead, 2016), entre otros términos. Para decirlo en una frase, si el problema de señalar la existencia de «autoritarismos subnacionales» era el estiramiento conceptual, las críticas derivaron en el problema también grave de la proliferación y confusión conceptual.

No obstante, algunas de las publicaciones más recientes han dedicado varias páginas, incluso capítulos enteros de libros, al tratamiento de los conceptos. Por ejemplo, los trabajos de Gervasoni (2011, 2016), Gibson (2013) y Giraudy (2015) no solo son explícitos en las definiciones conceptuales y operacionales sino que debaten sobre la pertinencia de las diferentes alternativas disponibles. Gervasoni (2010) argumenta que sería más pertinente considerar que se trata de «regímenes provinciales híbridos», porque son «similares a los que a nivel nacional han sido llamados 'democracias iliberales'” (Zakaria, 1997), 'autoritarismos competitivos' (Levitsky y Way, 2002) o 'autoritarismos electorales' (Schedler, 2006; Gervasoni, 2011, p. 78). En estos regímenes, según el autor, las elecciones multipartidarias y con voto universal son el principal medio para acceder al poder, pero los candidatos oficialistas superan masivamente a los opositores en cuanto a recursos de campańa y cobertura mediática, y respetan solo parcialmente sus libertades civiles al desplegar medidas represivas «blandas».

En un trabajo posterior, Gervasoni (2016) destaca que la diferencia entre regímenes autoritarios y democráticos son más evidentes a nivel nacional (por ejemplo, la distancia entre Corea del Norte y Finlandia), mientras que hay mayor cercanía entre los casos más democráticos y los menos democráticos a nivel subnacional (por ejemplo, entre San Luis y Mendoza en Argentina). Luego de un detallado desarrollo de la operacionalización del concepto, las fuentes de información y las reglas de agregación, el autor muestra resultados descriptivos donde incluso los casos peor situados en el índice son híbridos. Pero el uso del adjetivo «híbrido» como distintivo de un tipo de régimen, como han señalado Gibson (2013) y Giraudy (2015), adolece de precisión. En la literatura nacional estos regímenes incluyen «animales políticos» tanto autoritarios 
como democráticos 8 , por lo que resulta ser una etiqueta que puede desinformar acerca de las variaciones entre los casos.

Gibson opta por continuar con la denominación de «autoritarismos subnacionales», a partir del análisis del ejercicio efectivo de derechos políticos y no de las instituciones formales (2013, p. 14). Pero al no considerar la posibilidad de un continuum, el tipo de régimen político en este texto es más una cuestión de clases que de grados (Suárez-Cao, 2014). Al mismo tiempo, Gibson parece señalar que el adjetivo «subnacional» unido a la categoría "autoritarismo» implicaría inherentemente cierta hibridez, pero señalando en qué lado de la frontera (democrático o autoritario) se encuentran los casos. A pesar de este desarrollo conceptual, el problema con esta opción es que mantiene ambigüedad con respecto a la intensidad del concepto. El autor acepta que el fenómeno es bastante complejo y que existen casos más represivos que otros, más centrados en prácticas legales o más centrados en prácticas ilegales, y más o menos democráticos, incluyendo autoritarismos «puros». Pero el «autoritarismo subnacional» no captura esta variación.

El trabajo sobre México y Argentina de Giraudy (2015), en cambio, sí permite identificar diferentes tipos de regímenes políticos subnacionales. Al igual que Gervasoni, la autora plantea de forma detallada las definiciones conceptuales y operacionales, las fuentes de información y los procedimientos de agregación de dimensiones e indicadores. Encuentra, de esta manera, regímenes subnacionales no democráticos (subnational undemocratic regimes) en un extremo y regímenes democráticos en el otro, así como varios casos intermedios. Sin embargo, es criticable la formulación negativa del concepto que podría entenderse como «una categoría residual de todo lo que no es democrático (regímenes híbridos, autoritarios, totalitarios)»(Dosek, 2015, p. 658). Tampoco resulta claro por qué la autora no los califica como regímenes híbridos a pesar de que su definición — «regímenes civiles ni totalmente autoritarios ni totalmente democráticos» (Giraudy, 2015, p. 34) — es la que suele aplicarse para esta categoría. Según Dosek (2015), otro punto criticable es que la autora no seńala qué tan cercano a cero deben ser los valores para ser considerados no democráticos y que incluso los casos que obtienen cero no sean considerados autoritarios?. Es decir, elimina por definición la posibilidad del autoritarismo subnacional.

\footnotetext{
8 Por ejemplo, las democracias delegativas (O’Donnell 1994) y los autoritarismos competitivos (Levistky y Way, 2010). Véase también Collier y Levitsky (1997) y Linz (2000).

9 Sobre las decisiones de clasificaciones a partir de la data en publicaciones previas de la autora, véase Balán (2013).
} 
A pesar de estas discrepancias entre las opciones presentadas por los autores, todas tienen en común que toman como punto de partida alguna definición de democracia (si bien no la misma) y evalúan si los casos cumplen o no (o en qué medida) los criterios establecidos. Giraudy (2015) adopta una definición mínima (electoral) de la democracia, en la tradición de Przeworski, Alvarez, Cheibub y Limongi (1996), que subdivide en tres dimensiones: elecciones altamente disputadas (para el Ejecutivo y el Legislativo), elecciones limpias y alternancia en el poder. Por su parte, Gibson (2013) sigue la definición de la poliarquía de Dahl (1971) y las dos dimensiones que la componen (participación y oposición), distinguiendo si se están o no presentes en las unidades subnacionales. Gervasoni (2016), en cambio, parte de una definición «gruesa» que considera que la «democracia liberal representativa» está compuesta por dos dimensiones: la liberal (subdividida a su vez en derechos liberales y constreñimientos institucionales) y la democrática (oposición, efectividad e inclusión).

La importancia de este punto de partida es clave, como ilustra el caso de la región peruana de Áncash. Como demuestra el trabajo de Arévalo (2015), si se sigue la definición utilizada por Gibson, Áncash puede ser calificado como un autoritarismo subnacional que, además, no sería un autoritarismo puro en la medida que la autora argumenta que continuaron existiendo "arenas de lucha" como las señaladas para los regímenes nacionales (Levitsky y Way, 2002). La conceptualización de Gibson permite esta hibridez implícita. Por otro lado, Cueva et al. (2016) hacen uso de una definición procedimental de rango medio cercana a la de Gervasoni y, al mismo tiempo, utilizan una definición de autoritarismo que sí lo entiende desde la acepción «pura». Como consecuencia, los autores califican el caso como un tipo de régimen híbrido: un autoritarismo competitivo (Levitsky y Way, 2010) pero de nivel subnacional.

De lo mencionado hasta el momento se desprende que se trata de un debate en curso que permite evaluar las ventajas y desventajas de cada una de las alternativas. Lo importante es no perder de vista las diferencias en las definiciones y los procedimientos metodológicos desarrollados, porque las relaciones causales y las conclusiones a las que llegan los autores podrían diferir debido a las decisiones conceptuales previas. Incluso, como se plantea en la próxima sección, puede que no sean diferentes nombres utilizados para el mismo fenómeno, sino diferentes fenómenos que requieren distinguirse en las nomenclaturas con el fin de lograr refinamiento teórico. 


\section{LAS ESTRATEGIAS DESPLEGADAS POR LA LITERATURA SOBRE REGÍMENES POLÍTICOS NACIONALES CUANDO ENFRENTÓ RETOS CONCEPTUALES SIMILARES PUEDE BRINDAR MÚLTIPLES ENSEÑANZAS A LOS ESTUDIOS SOBRE REGÍMENES POLÍTICOS SUBNACIONALES}

Los debates conceptuales discutidos en la sección anterior son, en gran medida, herederos de las intricadas discusiones sobre qué es y qué no es la democracia en la literatura sobre regímenes políticos nacionales. Como es ampliamente conocido, la democracia es un concepto disputado (Held, 2006; Coppedge et al. 2011). En términos generales, existe un contraste entre las definiciones que podrían denominarse normativas, ideales o substantivas y aquellas que suelen ser catalogadas como empíricas, realistas o procedimentales. El primer grupo de definiciones suele enfocarse en determinados resultados como, por ejemplo, avances en la igualdad social; mientras que el segundo grupo trata de adherirse a consideraciones estrictamente políticas como un conjunto mínimo de procedimientos, dejando que las características económicas y sociales sean evaluadas como potenciales causas o consecuencias (Collier y Levitsky, 1997: 433). Aquellos que trabajan en la subdisciplina de la política comparada suelen emplear mayoritariamente el segundo grupo de definiciones, sea por su utilidad analítica y/o porque consideran que una definición demasiado ambiciosa podría hacerle el juego al autoritarismo al despreciar la democracia realmente existente (O'Donnell, 2007). Sin embargo, como demuestran las diferentes definiciones procedimentales que adaptan Giraudy, Gibson y Gervasoni del ámbito nacional al subnacional, en este segundo grupo también existen diferencias, generando retos para la acumulación de conocimiento: los autores no quieren decir lo mismo cuando plantean que un caso es democrático y otro no.

La ola global de cambios de regímenes políticos mencionada previamente generó retos adicionales para los estudios centrados en la democracia nacional. Surgieron nuevos regímenes políticos alrededor del mundo que si bien compartían algunos atributos de la democracia, también diferían mucho entre sí y del conjunto relativamente pequeño de democracias tradicionales en los países económicamente más avanzados. Como destacaron hace dos décadas Collier y Levitsky (1997), esto generó que proliferaran cientos de nuevos e innovadores conceptos que hacían referencia a democracias "con adjetivos», pero con el riesgo del estiramiento y la confusión conceptual. Como consecuencia, si bien la conceptualización de regímenes políticos subnacionales tiene sus propios retos (a saber, la importancia de capturar la particularidad de la relación entre los diferentes niveles de gobierno y los constreńimientos que impone el centro 
político), otros se relacionan con problemáticas por las que ya han transitado sus antecesores nacionales. Por lo tanto, los estudios subnacionales harían bien en reconocer no solo estos retos comunes sino también las estrategias desarrolladas previamente.

En primer lugar, cuando en la literatura nacional se amplió el conjunto de casos bajo análisis, no se negó por definición la existencia de algún tipo de régimen, sino que la evidencia guió las clasificaciones. En la literatura subnacional, existen razones de peso para seguir por esta senda. Plantear teóricamente que no podrían existir autoritarismos subnacionales no parece ser el mejor camino, porque la respuesta debería ser empírica. Establecidos los criterios para que los casos puedan llevar esta denominación, se podrá evaluar si un caso concreto es o no un autoritarismo subnacional. En cambio, si los conceptos y las herramientas de medición están construidos de tal manera de que no existe esta posibilidad, ningún caso será un autoritarismo subnacional sin importar la evidencia que se recolecte.

Lo mismo aplica para la diferencia que se hace entre unidades subnacionales en países federales y unitarios, sobre todo porque esta opción previene de encontrar relaciones causales. ¿Cómo podría afirmarse que los países unitarios no tienen regímenes que se desvían de las características democráticas debido a carecer de las instituciones formales de los países federales, si no tener estas instituciones es lo que en primer lugar llevó a considerar que no tienen este tipo de régimen? Precisamente, es a partir de criterios que aplican más allá de que los casos pertenezcan o no a países federales, que se ha documentado este fenómeno en países unitarios como Filipinas (Sidel, 2014), Colombia (Eaton y Prieto, en prensa) y Perú (Arévalo, 2015; Cueva et al., 2016).

En segundo lugar, así como la literatura sobre regímenes políticos subnacionales no vio la necesidad de subsumir los nuevos casos bajo una sola rúbrica, no resulta forzoso hacerlo en los estudios subnacionales. Lo más probable es que estemos no ante uno, sino varios fenómenos. En un trabajo de revisión de literatura, Balán (2013) resaltaba que en algunas provincias argentinas no se encuentran siquiera los atributos electorales de la democracia, en la medida que los gobernadores eran elegidos con más de $70 \%$ de los votos y en algunas provincias (como San Luis en 2003) la cifra era de casi 100\%. Pero el sólido sur en Estados Unidos entre fines del siglo XIX e inicios del siglo XX provee el caso más extremo de privación de derechos en la historia de la democracia (Gibson, 2013 , p. 3). Estos casos difícilmente podrían ser entendidos como regímenes híbridos y se acercan más al tipo ideal de regímenes autoritarios. 
Otros casos, la mayoría de ellos, son regímenes híbridos, porque «no todas las provincias o estados que no cumplen los estándares democráticos constituyen regímenes totalmente autoritarios [...]» (Behrend y Whitehead, 2016, p. 292). Así, una enseñanza adicional de la literatura nacional, como se mencionó previamente, es que conviene identificar si la hibridez cruza la línea del autoritarismo o no. Por ejemplo, si se trata de democracias delegativas (O’Donnell, 1994) o autoritarismos competitivos (Levitsky y Way, 2010). Finalmente, el reciente trabajo de Behrend y Whitehead expande el campo de estudio de los regímenes políticos a las prácticas y estructuras iliberales que pueden «restringir las alternativas, limitar el debate, excluir o distorsionar la participación democrática de tal manera que [...] puede disminuir severamente el estatus democrático particular de una provincia o jurisdicciones subnacionales sin manifestar ampliamente la totalidad de características de los regímenes autoritarios» (2016, p. 5). Los autores nos recuerdan que un régimen político requiere sistematicidad.

Para ilustrar este punto, se puede considerar nuevamente el contraste entre algunas regiones peruanas. Como motivo de un libro en coautoría junto a Noelia Chávez y Carla Cueva, condujimos un trabajo de campo en Áncash y Loreto que nos permitió demostrar cierto paralelo entre César Álvarez, presidente regional de Áncash, e Iván Vásquez, presidente regional de Loreto. Ambos fueron elegidos en 2006, contaron con niveles altos de aprobación popular, lograron reelegirse en 2010 y tuvieron una clara vocación de permanecer en el cargo. Vásquez también actuó con gran discrecionalidad durante estos años y fue involucrado en diversos casos de corrupción (la compra irregular de «motochatas», el escándalo sobre los créditos agrarios, la obra de alcantarillado, etcétera) que lo llevaron a la cárcel. Asimismo, los testimonios revelaron una relación conflictiva con la prensa y su intento de controlar organizaciones de la sociedad civil que le hacían contrapeso. Sin embargo, la diferencia con Áncash es la gravedad y la recurrencia de estos hechos que son, básicamente, casos aislados. Los políticos opositores, como el consejero regional Quispe Farro, pudieron cumplir sus funciones sin que su vida corriera peligro. Tampoco se encontró un clima de miedo y censura sistemática como en Áncash, porque los periodistas críticos eran numerosos y continuaron realizando sus labores, aunque ello implicara cambiar de medio. De la misma manera, el Frente Patriótico logró resistir el intento de cooptación de Vásquez entre 2011 y 2012, y retomó su autonomía luego de este periodo. En Loreto no se encuentra nada parecido a una central de inteligencia como "La Centralita», que permitió desarrollar prácticas autoritarias sistemáticas. En suma, pese las similitudes, no ocurrió 
un cambio de régimen en Loreto y la democracia se mantuvo. Existieron, en lenguaje de Behrend y Whitehead, prácticas iliberales que no configuraban un autoritarismo. Ni siquiera, como se argumenta en el libro, un autoritarismo competitivo como sí califica Áncash.

Finalmente, vale la pena recordar que la literatura nacional desarrolló cinco estrategias conceptuales: diferenciar características, evitar el estiramiento conceptual, crear subtipos disminuidos, precisar el concepto de democracia y cambiar el concepto de aplicación (Collier y Levistsky, 1997). Todas se encuentran relacionadas con la escala de abstracción de Sartori (1970), que plantea que «a medida que un concepto aumenta su connotación, es decir, el número de características y propiedades que lo definen, disminuye su denotación, ya que hay un número de objetos o eventos menor que se ajusta a ese conjunto de propiedades» (Anduiza, Crespo y Méndez, 1999, p. 41).

De esta manera, la diferenciación consiste en moverse hacia abajo en la escala de abstracción, aumentando atributos y reduciendo la extensión de casos que abarca el concepto, como sucede al crear subtipos de regímenes como "democracia parlamentaria», "democracia federal», "democracia multipartidista» (Collier y Levitsky, 1997, p. 435). Para evitar el estiramiento conceptual, los autores también se movieron hacia arriba en la escala de abstracción, reduciendo los atributos y aumentando la extensión de casos que puede contener el concepto; por ejemplo, los "regímenes civiles», "regímenes competitivos» y «regímenes electorales» no son necesariamente democráticos (p. 436). Por otra parte, los subtipos disminuidos permiten lograr ambos objetivos de forma simultánea, sin subir o bajar en el nivel de abstracción, porque los autores plantean conceptos que destacan que los casos no son plenamente democráticos, pero resaltando qué atributos de la democracia permanecen o faltan. Por ejemplo, cuando se plantea que un caso es una «democracia electoral» o una «democracia iliberal»se está resaltando que la amplia protección de derechos civiles no está presente; mientras que cuando se plantea que un caso es una «democracia oligárquica», tiene como dimensión faltante el sufragio universal (p. 441) ${ }^{10}$. La estrategia de precisar el concepto de democracia implica revisar la definición e incluir nuevos atributos que eran dados por sentado antes de que se aplicaran a nuevos contextos; por ejemplo, al establecerse que se requiere que las autoridades elegidas gobiernen efectivamente (p. 443). Por último, cambiar el concepto al que aplica puede reducir el estándar democrático — por ejemplo, cuando se plantea una «situación» o un «gobierno» autoritario o democrático-

10 De la misma manera, se han planteado autoritarismos «con adjetivos». 
o puede aumentarlo — por ejemplo, cuando se señala la idea de un «estado» democrático o autoritario (p. 446), pero sin recurrir a modificar la definición de régimen democrático-.

En el estudio de los regímenes políticos subnacionales, algunas de estas estrategias han sido aplicadas. El concepto de autoritarismo subnacional de Gibson (2013) que señala una hibridez implícita, parece seguir la estrategia de los subtipos disminuidos. Giraudy (2015) y Gervasoni (2016), en cambio, evitan el estiramiento conceptual colocándose en un nivel de abstracción mayor señalando la existencia de regímenes no democráticos y regímenes híbridos, respectivamente. Behrend y Whitehead (2016) seguirían, más bien, la estrategia de cambiar el concepto al que se aplica la denominación: son iliberales las prácticas y no los regímenes. Cambiar el concepto de aplicación también fue propuesto previamente por Behrend (2012) al distinguir entre prácticas, situaciones y regímenes autoritarios, según el grado de vulneración de las dimensiones de la democracia.

\section{HASTA EL MOMENTO NO EXISTE UNA TEORÍA GENERAL SOBRE REGÍMENES POLÍTICOS SUBNACIONALES Y ALGUNAS DE LAS EXPLICACIONES MÁS RECURRENTES EN LA LITERATURA TIENEN LIMITACIONES PARA APLICAR A JuRisdicciones dentro de Países unitarios Como el Perú}

Como indica Balán, «hasta la fecha, no existe una teoría general del autoritarismo subnacional» (2013, p. 7). Las discrepancias entre los autores, entonces, no se limitan al ámbito conceptual sino también al explicativo. Existen diferentes maneras de clasificar las causas señaladas por los autores, incluyendo distinguir entre explicaciones estructurales y estratégicas (Balán, 2013, p. 7) o entre causas institucionales, económicas, sociológicas y partidarias (Behrend y Whitehead, 2016, p. 158). Sin embargo, siguiendo a Cueva et al. (2016), también puede diferenciarse entre explicaciones basadas en factores exógenos de aquellas enfocadas en factores endógenos, sin que esto prevenga de resaltar las aproximaciones teóricas tomadas en consideración. En gran medida, este ha sido el principal flanco de discrepancia, porque «los trabajos existentes [...] generalmente han enfatizado factores subnacionales o la interacción nacionalsubnacional» (Giraudy, 2015, p. 15).

Las explicaciones endógenas suelen tomar como punto de partida la teoría de la modernización. El trabajo de McMann (2006) explica la democratización subnacional en Rusia y Kirguistán a partir del desarrollo capitalista, considerando 
que la autonomía política permite a la ciudadanía involucrarse en política y desafiar a los gobernantes (Giraudy, 2015, p. 15). Montero (2007) hace un argumento similar para Brasil, señalando que «las variables político-económicas pueden tener más peso en democracias donde los partidos nacionales son débiles y proveen escasa continuidad o vínculos funcionales entre titulares subnacionales y élites nacionales» $(2007$, p. 6). En un trabajo posterior, el autor identifica otras variables relacionadas a los niveles de modernización: los lugares con poblaciones pequeñas, altos niveles de pobreza y deficiente comunicación con los centros urbanos hacen posible que la población se involucre en redes clientelistas que atentan contra la democracia subnacional (Montero, 2011). Este argumento fue trabajado explícitamente por Montero debido a la debilidad de los partidos políticos en Brasil en comparación a Argentina y México.

Otro tipo de explicaciones centradas en factores internos de las jurisdicciones subnacionales se distingue de las mencionadas. El análisis de Durazo Herrmann (2010) sobre el estado de Oaxaca en México resalta que el autoritarismo subnacional continuó pese a la democratización nacional debido al mantenimiento de estructuras sociales patrimonialistas. En cambio, para explicar la particularidad de las provincias argentinas de San Luis y Corrientes, Behrend (2011) desarrolla el concepto de juegos cerrados donde la oposición no es una fuerza democratizadora, sino que busca reemplazar a las familias gobernantes en la perpetuación del poder. Estos juegos cerrados «suelen surgir en provincias con una estructura económica limitada, población pequeña, y oportunidades de negocios limitadas. Las élites políticas familiares usan el Estado para promover sus intereses económicos y controlar el acceso a actividades empresariales [...]»(2011, p. 154). Mientras que, para casos fuera de América Latina, Lankina (2012) argumenta que «el capital humano precomunista afecta las variaciones en el capital humano en la actualidad y en la democracia de las regiones de Rusia. Ella encuentra que la educación pre-comunista es un predictor de la modernización postcomunista» (Giraudy, 2015, p. 16). Es decir, si bien importa la modernización, esta depende del legado de regímenes políticos autoritarios previos.

No obstante, las explicaciones con mayor aceptación en la literatura han sido aquellas que hacen referencia a factores exógenos o, mejor dicho, a la relación nacional-subnacional. Estos argumentos hacen énfasis en que las unidades subnacionales no son jurisdicciones autónomas e independientes de la política nacional (Giraudy, 2015, p. 16), sino subsistemas de un sistema mayor: su relación con la política nacional es de "parte-todo». Plantean que lo más apropiado es estudiar estos fenómenos a partir de teorías de política 
territorial que, estrictamente, no son sobre el territorio sino sobre el modo en que la organización y la lucha por la política se desarrollan a lo largo del territorio (Gibson, 2013, p. 15).

Una de sus versiones más influyentes es la del libro de Gibson (2013) —aunque fue trabajada en publicaciones previas - y su propuesta sobre el «control de fronteras». De acuerdo con este trabajo, los autoritarismos subnacionales prevalecen allí donde sus gobernantes son capaces de limitar el alcance de los conflictos de su jurisdicción, evitando que adquieran carácter nacional y/o que las fuerzas opositoras locales accedan a aliados y recursos nacionales. Las estrategias que despliegan los gobernantes autoritarios incluyen diseñar las constituciones locales y las reglas electorales, la influencia sobre los partidos políticos y la legislatura nacional a través de los parlamentarios que responden a ellos, y el control de la agenda legislativa en asuntos que afectan su jurisdicción (Gibson, 2013, pp. 26-30). Gibson, asimismo, enfatiza la importancia de las instituciones políticas de organización territorial: el régimen territorial de un país. Este:

[...] podrá ser más o menos descentralizado, según su nivel de fragmentación vertical, y más o menos asimétrico, según su nivel de fragmentación horizontal. El cruce de las dos dimensiones permite distinguir que los regímenes territoriales descentralizados y asimétricos son más periféricos, a diferencia de los centralizados y simétricos (Suárez-Cao, 2014, p. 193).

En los regímenes más periféricos, los autoritarismos subnacionales se configuran a través de reglas formales; mientras que en regímenes menos periféricos, los gobernantes se apoyan en reglas informales.

La propuesta de Gervasoni (2010), por contraste, ha puesto énfasis en la importancia de las instituciones y la economía política para el mantenimiento de los autoritarismos subnacionales. Luego de mostrar que las transferencias intergubernamentales actúan en el ámbito subnacional como lo hacen las rentas de recursos en el nivel nacional, el autor encuentra que «mayores transferencias por adulto del gobierno federal están asociadas con menos democracias» y que estos efectos son «altamente significativos y substancialmente importantes» (Gervasoni, 2010, p. 321). Asimismo, evidencia que los mecanismos causales son muy cercanos a los «efectos en el gasto» señalados por Goldberg, Wibbels y Mvukiyehe (2008); es decir, una dependencia económica hacia el estado subnacional que merma la posibilidad de plantear demandas de la ciudadanía y parcializa a los medios de comunicación.

Giraudy (2015), por su parte, tiene la particularidad de adherirse a las explicaciones exógenas y a la vez resaltar la variedad de características 
particulares de las unidades subnacionales. La autora explica la continuidad de los regímenes no democráticos subnacionales a partir de una teoría compleja que considera los instrumentos de control del presidente sobre los gobernadores (fiscal y partidario) y los de autonomía de los gobernadores frente al presidente (financiero y de administración estatal). A partir de estos, es posible que existan dos rutas para la continuidad de estos regímenes. Primero, puede haber una «reproducción desde arriba» cuando los presidentes, movidos por sus intereses políticos en el nivel nacional, ejercen poder sobre los gobernadores de provincias no democráticas. Segundo, la «reproducción desde abajo» ocurre cuando los presidentes no tienen suficiente poder y/o los gobernadores pueden neutralizarlos y lograr su supervivencia a través de medios propios.

Aunque estas explicaciones fueron concebidas para entender los regímenes políticos subnacionales en países federales, también pueden ayudar a entender lo que ocurre en países unitarios. Eaton y Prieto (en prensa), sobre Colombia, plantean que «las élites subnacionales en países unitarios pueden no disfrutar del rango de prerrogativas disponibles para sus pares en países federales, pero esta diferencia institucional no previene que terminen esencialmente haciendo uso de las mismas tres estrategias de control de fronteras identificadas por Gibson", si bien los niveles de violencia y la relación con grupos paramilitares no tiene paralelo con lo ocurrido en Argentina, México y Brasil. Arévalo también analiza lo sucedido en Perú con base en la teoría de Gibson, mencionando que «el gobierno central necesitaba que Álvarez mantenga el "orden político", provea de servicios a la región y —a futuro- brinde réditos políticos en forma de votos» $(2015$, p. 31). La autora documenta los vínculos con partidos políticos nacionales, congresistas, el exfiscal de la Nación y el gobierno central de Ollanta Humala, destacando que durante un largo periodo César Álvarez logró un efectivo control de fronteras.

No obstante, también se deberá tomar en cuenta las limitaciones de estas teorías para viajar a nuevos contextos. Particularmente en Perú, el autoritarismo subnacional no proviene de periodos predemocráticos, sino que emergió luego de la transición a la democracia de 2001 y la descentralización de 2002. Así, la pregunta centrada en la estabilidad es importante, pero también entender los orígenes de la caída de la democracia en Áncash. Por otro lado, Perú se distingue de sus pares federales no solo en la debilidad de los partidos políticos y su dificultad para competir en arenas subnacionales (Zavaleta, 2014; Seifert, 2014), sino también en que los actores regionales no han alcanzado relevancia nacional (Dargent y Muñoz, 2012). En este sentido, pese a que existió el vínculo con el nivel nacional, como demuestra Arévalo, este podría 
haber operado de modo diferente: la capacidad de los gobernantes subnacionales para brindar apoyo electoral y gobernabilidad al nivel nacional parece limitado en un país con las características del Perú (Cueva et al., 2016). De la misma manera, para explicar por qué ocurrió este fenómeno en Áncash se requiere entender por qué no ocurrió en otras regiones, por lo que se requerirá realizar este ejercicio comparativo en el futuro. Finalmente, los casos donde no ocurrió un autoritarismo podrían variar en sus niveles de afectación a las dimensiones de la democracia o podría encontrarse que existen distintos tipos de democracias subnacionales. Otra agenda de investigación pendiente apunta a diferenciar entre democracias.

En suma, queda claro que todavía es mucho lo que debe ser investigado en este campo de estudio. Al ampliarse el conjunto de casos donde se encuentra el fenómeno, resulta necesario revisar las explicaciones que servían para dar cuenta de la estabilidad de los autoritarismos subnacionales en los federalismos. No obstante, también deberá teorizarse sobre otros fenómenos que antes no han sido centrales. Particularmente, los orígenes y la caída de los autoritarismos subnacionales, así como las variaciones en los niveles de democracia o en las tipologías de democracia que se establezcan.

\section{BibLIOGRAFÍA}

Almond, G. A. y Verba, S. (1963). The Civic Culture: PoliticalAttitudes and Democracy in Five Nations. Princeton, NJ: Princeton University Press. doi: https://doi.org/10.1515/9781400874569

Anduiza, E. , Crespo, I. y Méndez, M. (1999). Cuadernos Metodológicos: Metodología de la Ciencia Política. Madrid, España: Centro de Investigaciones Sociológicas.

Arévalo, R. (2015). La dinámica politica a nivel subnacional: la estabilidad en la gestión de César Álvarez (2006-2013). Tesis de licenciatura en Ciencia Política y Gobierno. Pontificia Universidad Católica del Perú. Lima, Perú.

Balán, P. E. (noviembre de 2013). Democracia y representación. Autoritarismo subnacional: clasificación, causas, teoría. En VII Jornadas de Jóvenes Investigadores. Instituto de Investigaciones Gino Germani, Facultad de Ciencias Sociales de la UBA. Buenos Aires, Argentina.

Behrend, J. (2011). The Unevenness of Democracy at the Subnational Level: Provincial Closed Games in Argentina. Latin American Research Review, 46(1), 150-176.

Behrend, J. (2012). Democratización subnacional: Algunas preguntas teóricas. POSTData, 17(2), 11-34. doi: 10.1353/lar.2011.0013

Behrend, J. y Whitehead, L. (eds.) (2016a). The struggle of subnational democracy. Journal of democracy, 27(2), 155-167. doi: 10.1353/jod.2016.0023

Behrend, J. y Whithead, L. (eds.) (2016). Illiberal Practices: Territorial variance in large federal democracies. Baltimore, MD: The John Hopkins University Press.

Benton, A. L. (2012). Bottom-Up Challenges to National Democracy. México's (Legal) Subnational Authoritarian Enclaves. Comparative Politics, 44(3), 253-271. doi: $10.5129 / 001041512800078931$ 
Collier, D. y Levitsky S. (1997). Democracy with adjectives: Conceptual Innovation in Comparative Research. World Politics, 49(3), 430-451. doi: 10.1353/wp.1997.0009

Coppedge, M., Gerring, J., Altman, D., Bernhard, M., Fish, S., Hicken, A., ..., Teorell, J. (2011). Conceptualizing and Measuring Democracy: A New Approach. Perspectives on politics, 9(2), 247-267. doi: 10.1017/S1537592711000880

Cueva, C., Chávez, N., Encinas, D. y Ramírez, M. (2016). Democracia y territorio en países unitarios: una agenda de investigación pendiente. Lima: Fondo Editorial del Jurado Nacional de Elecciones.

Dahl, R. A. (1971). Polyarchy. Participation and Opposition. New Haven, CT: Yale University Press.

Dargent, E. y Muñoz, P. (2012). Perú 2011: Continuidades y cambios en la política sin partidos. Revista de Ciencia Politica, 2(1), 245-268. doi: 10.4067/S0718-090X2012000100013

Došek, T. (2015). [Reseña del libro Democrats and Autocrats. Pathways of Subnational Undemocratic Regime Continuity within Democratic Countries, de Agustina Giraudy]. Revista de Ciencia Política, 35(3), 655-660.

Durazo Herrmann, J. (2010). Neo-Patrimonialism and Subnational Authoritarianism in Mexico. The Case of Oaxaca. Journal of Politics in Latin America, 2(2), 85-112.

Eaton, K. y Prieto J. D. (en prensa 2017). Subnational authoritarianism in Colombia: Divergent Paths in Cesar and Magdalena. En T. Hilgers y L. Macdonald (eds.), Violence in Latin America and the Caribbean (pp. 153-172). Nueva York, NY: Cambridge University Press.

Encinas, D., Chávez, N. y Cueva, C. (en elaboración). Autoritarismos subnacionales en países unitarios: recursos, crimen y poder en el Perú subnacional. (Manuscito inédito).

Escobar-Lemmon, M. (2001). Fiscal Decentralization and Federalism in Latin America. Publius, 31(4), 23-41. doi: 10.1093/oxfordjournals.pubjof.a004919

Falleti, T. G. (2005). A Sequential Theory of Decentralization: Latin American Cases in Comparative Perspective: Argentina and Colombia. American Political Science Review, 99(3), 327-346. doi: $10.1017 /$ S0003055405051695

Fox, J. (1994). The Difficult Transition from Clientelism to Citizenship: Lessons from Mexico. World Politics, 46(2), 151-184. doi: 10.2307/2950671

Geddes, B. (1999). What do we know about democratization after twenty years? Anual Review of Political Science, 2, 115-144. doi: 10.1146/annurev.polisci.2.1.115

Gervasoni, C. (2010). A Rentier Theory of Subnational Regimes: Fiscal Federalism, Democracy, and Authoritarianism in the Argentine Provinces. World Politics, 62(2), 302-340. doi: 10.1017/ s0043887110000067

Gervasoni, C. (2011). Democracia, autoritarismo e hibridez en las provincias argentinas: La medición y causas de los regímenes subnacionales. Journal of Democracy en español, 3, 75-93.

Gervasoni, C. (2016). The dimensions of democratic and hybrid subnational regimes: evidence from an expert survey in Argentina. En J. Behrend y L. Whitehead (eds.), Illiberal Practices: Territorial variance in large federal democracies (pp. 120-161). Baltimore, MD: The Johns Hopkins University Press.

Gibson, E. L. (2005). Boundary Control: Subnational Authoritarianism in Democratic Countries. World Politics, 58(1), 101-132. doi: 10.1353/wp.2006.0018

Gibson, E. L. (2013). Boundary Control: Subnational Authoritarianism in Federal Democracies. Cambridge, MA: Cambridge University Press, Cambridge Studies in Comparative Politics Series.

Gibson, E. L. (ed.) (2004). Federalism and Democracy in Latin America. Baltimore, MD: The Johns Hopkins University Press.

Giraudy, A. (2009). Subnational undemocratic regime continuity after democratization: Argentina and Mexico in comparative perspective. Chapel Hill, NC: University of North Carolina at Chapel Hill (Manuscrito). 
Giraudy, A. (2012). Subnational Democracy: Lessons from Latin America. APSA Comparative Democratization Newsletter, 10(1), 1-8. doi: 10.1093/acprof:oso/9780198706861.001.0001

Giraudy, A. (2015). Democrats and Autocrats. Pathways of Subnational Undemocratic Regime Continuity within Democratic Countries. Oxford, Inglaterra: Oxford University Press.

Goldberg, E., Wibbels, E. y Mvukiyehe, E. (2008). Lessons from Strange Cases: Democracy, Development, and the Resource Curse in the U.S. States. Comparative Political Studies, 41(4-5), 477-514. doi: 10.1177/0010414007313123

Hagopian, F. (1996). Traditional Politics and Regime Change in Brazil. doi: 10.1017/CBO9780511584862 Held, D. (2006). Models of Democracy, 3rd ed. Cambridge, MA: Polity Press.

Helmke, G. y Levitsky, S. (eds.). (2006). Informal Institutions and Democracy. Lessons from Latin America. Baltimore, MD: The Johns Hopkins University Press.

Key, V. O. (1949). Southern Politics in State and Nation. Nueva York, NY: Alfred A. Knopf Publisher.

Lankina, T. V. (2012) Religious influences on human capital variations in imperial Russia. Journal of Eurasian Studies, 3(1), 10-19. doi: 10.1016/j.euras.2011.10.002

Levitsky, S. y Way, L. (2002). The Rise of Competitive Authoritarianism. Elections Without Democracy. Journal of Democracy, 13(2), 51-65. doi: 10.1353/jod.2002.0026

Levitsky, S. y Way, L. (2010). Competitive Authoritarianism: Hybrid Regimes after the Cold War. doi: $10.1017 / \mathrm{CBO} 9780511781353$

Levitsky, S. y Way, L. (2015). The myth of democratic recession. Journal of Democracy, 26(1), 45-58.

Linz, J. J (1964). An Authoritarian Regime: The Case of Spain. En E. Allard y Y. Littunen (eds.), Cleavages, Ideologies and Party Systems (pp. 129-148). Helsinki, Finlandia: Academic Bookstore.

Linz, J. J. (2000). Totalitarian and authoritarian regimes. Boulder, CO: Lynne Rienner.

Lipset, S. M. (1960). Political Man: The Social Bases of Politics. Garden City, NY: Doubleday \& Company.

McMann, K. (2006). Economic Autonomy and Democracy: Hybrid Regimes in Russia and Kyrgyzstan. Cambridge, Inglaterra: Cambridge University Press. doi: 10.1017/CBO9780511510281

Montecinos, E. (2007). Limitaciones del diseño institucional para una gestión municipal participativa. El caso chileno. Economía, Sociedad y Territorio. Santiago, 5(23), 725-743. doi: 10.22136/est002007256

Montero, A. P. (2007). Uneven Democracy? Subnational Authoritarianism in Democratic Brazil. En Latin American Studies Association Annual Meeting. Llevado a cabo en Montreal, Quebec, Canadá.

Montero, A. P. (2010). No Country for Leftists? Clientelist Continuity and the 2006 Vote in the Brazilian Northeast. Journal of Politics in Latin America, 2(2), 113-153.

Montero, A. P. (2011). The New Boss Same as the Old Boss? Incumbency and the Decline of Conservative Rule in the Brazilian Northeast. APSA 2011 Annual Meeting Paper. Recuperado de: https://papers. ssrn.com/sol3/papers.cfm?abstract_id=1901982

Moore, B. (1966). Social Origins of Dictatorship and Democracy: Lord and Peasant in the Making of the Modern World. Boston, MA: Beacon Press.

O’Donnell, G. (1993). Acerca del Estado, democratización y algunos problemas conceptuales. Desarrollo Económico, 33(130), 1-32. doi: 10.2307/3467251

O’Donnell, G. (1994). Delegative Democracy. Journal of Democracy, 5(1), 55-69. doi: 10.1353/ jod.1994.0010

O’Donnell, G. (1996). Illusion about Consolidation. Journal of Democracy, 7(2), 34-51. doi: 10.1353/ jod.1996.0034

O'Donnell, G. (2007). Disonancias. Buenos Aires, Argentina: Prometeo.

O’Donnell, G. y Schmitter, P. C. (1986). Transitions from Authoritarian Rule. Baltimore, MD: The Johns Hopkins University Press, The Woodrow Wilson International Center for Scholars.

Przeworski, A., Alvarez, M., Cheibub, J. L. y Limongi, F. (1996). What Makes Democracy Endure? Journal of Democracy, 7(1), 39-55. doi: 10.1353/jod.1996.0016 
Rebolledo, J. (2011). Black Sheep of the Family: A Model of Subnational Authoritarian Endurance in National Democracies. New Haven, CT: Yale University Press.

Sartori, G. (1970). Concept Misformation in Comparative Politics. American Political Science Review, 64(4), 1033-1053. doi: 10.2307/1958356

Schedler, A. (2006). The Logic of Electoral Authoritarianism. En A. Schedler (ed.), Electoral Authoritarianism. The Dynamics of Unfree Competition (pp. 3-33). Boulder, CO: Lynne Rienner.

Seifert Bonifaz, M. (2014). Colapso de los partidos nacionales y auge de los partidos regionales. Lima: Escuela de Gobierno y Políticas Públicas de la Pontificia Universidad Católica del Perú.

Sidel, J. T. (2014). Economic foundations of subnational authoritarianism. Democratization, 21, 161 184. doi: 10.1080/13510347.2012.725725

Snyder, R. (1999). After the States Withdraws: Neoliberalism and Subnational Authoritarian Regimes in Mexico. En W. A. Cornelius, T. A. Eisenstadt y J. Hindley (eds.), Subnational Politics and Democratization in Mexico (pp. 259-341). San Diego, CA: Center for US-Mexican Studies, University of California Press.

Snyder, R. (2001). Scaling Down: The Subnational Comparative Method. Studies in Comparative International Development, 36(1), 93-110. doi: 10.1007/BF02687586

Snyder, R., Moncada, E. y Giraudy, A. (en prensa). Subnational Research in Comparative Politics: Achievements and Prospects. (Manuscrito inédito).

Suárez-Cao, J. (2014). [Reseña] Boundary control: subnational authoritarianism in Federal democracies. América Latina Hoy, 66, 192-194.

Tunón, G. (2007). Democratización subnacional: una exploración a la luz del caso argentino. (Tesis de Licenciatura). Universidad de San Andrés, Buenos Aires, Argentina,

Zakaria, F. (1997). The Rise of Illiberal Democracy. Foreign Affairs, 76(6), 22-41. doi: 10.2307/20048274

Zavaleta, M. (2014). Coaliciones de independientes: las reglas no escritas de la politica electoral. Lima: Instituto de Estudios Peruanos. 